\title{
Rapid optimization of gene dosage in E. coli using DIAL strains
}

\author{
Joshua T Kittleson', Sherine Cheung ${ }^{2}$ and J Christopher Anderson ${ }^{1,3^{*}}$
}

\begin{abstract}
Background: Engineers frequently vary design parameters to optimize the behaviour of a system. However, synthetic biologists lack the tools to rapidly explore a critical design parameter, gene expression level, and have no means of systematically varying the dosage of an entire genetic circuit. As a step toward overcoming this shortfall, we have developed a technology that enables the same plasmid to be maintained at different copy numbers in a set of closely related cells. This provides a rapid method for exploring gene or cassette dosage effects.

Results: We engineered two sets of strains to constitutively provide a trans-acting replication factor, either Pi of the R6K plasmid or RepA of the ColE2 plasmid, at different doses. Each DIAL (different allele) strain supports the replication of a corresponding plasmid at a constant level between 1 and 250 copies per cell. The plasmids exhibit cell-to-cell variability comparable to other popular replicons, but with improved stability. Since the origins are orthogonal, both replication factors can be incorporated into the same cell. We demonstrate the utility of these strains by rapidly assessing the optimal expression level of a model biosynthetic pathway for violecein.

Conclusions: The DIAL strains can rapidly optimize single gene expression levels, help balance expression of functionally coupled genetic elements, improve investigation of gene and circuit dosage effects, and enable faster development of metabolic pathways.
\end{abstract}

\section{Background}

Optimizing desired outcomes by varying a design parameter is a staple of almost every engineering field, from mechanical engineers tweaking blade angles on a wind turbine to civil engineers altering the timing of traffic lights. Similarly, genetic engineers alter gene expression levels to optimize some desirable phenotype. Strong overproduction of single proteins can impose a metabolic burden on $E$. coli, and often a lower expression level leads to improved phenotype [1]. In multi-subunit proteins and genetic circuits, expression of particular proteins often needs to be balanced for proper function (e.g. [2,3], and [4]). Extensive work has established methods for achieving expression of a gene or operon at a particular level, including control of transcription using standard promoter sets [5], modulation of RNA processing [6], and control of translation through ribosome binding site (RBS) manipulation [7]. However, using these tools to investigate the desired expression

\footnotetext{
* Correspondence: jcanderson@berkeley.edu

'Department of Bioengineering, University of California, Berkeley, USA

Full list of author information is available at the end of the article
}

level of a single gene or operon requires cloning for each level to be tested. Using inducible promoter systems to probe multiple expression levels can rapidly determine an approximate desired expression level, but does not provide a genetically encoded solution, which can be useful for downstream applications. For optimizing multi-operon constructs, fewer tools exist. Generating large numbers of repeats in the genome is labor intensive [8], while strategies that increase plasmid copy number upon induction provide a narrow range of copy numbers [9], cause runaway replication $[10,11]$, or are incompatible with constructs using the common $\mathrm{P}_{\mathrm{BAD}}$ promoter [12]. To address these shortcomings and allow researchers to explore the effect of copy number on genetic devices, we have exploited an underutilized control point: plasmid copy number.

Genetic engineers working in E. coli are blessed with a wide range of plasmid systems and plasmid copy numbers to choose from, ranging from single copy BACs to $\sim 500$ copy pUC plasmids [13]. However, to take advantage of copy number differences, each gene or device of interest has to be cloned into different plasmids,
C Biomed Central

() 2011 Kittleson et al; licensee BioMed Central Ltd. This is an Open Access article distributed under the terms of the Creative Commons Attribution License (http://creativecommons.org/licenses/by/2.0), which permits unrestricted use, distribution, and reproduction in any medium, provided the original work is properly cited. 
necessarily changing the local genetic context along with the copy number. A notable exception to this rule is the gamma origin of the R6K plasmid, which requires the trans-acting Pi protein to initiate replication [14]. Different alleles of pir integrated into the $E$. coli genome are known to support R6K plasmids at different copy numbers (e.g. pirt and pir116 [15]), meaning that the genetic context on the plasmid itself remains unaltered. Similarly, the orthogonal replicon of ColE2-P9 also uses a trans-acting factor, RepA, to support the origin of replication [16].

In this work, we generate two sets of strains bearing different alleles (DIAL) of pir or repA to support the same plasmid at a wide range of copy numbers. We then characterize the copy number, cell-to-cell variability, and stability of plasmids in both sets of DIAL strains. We illustrate their utility on a model system by examining expression of the violacein biosynthesis pathway [17] at different copy numbers. The results demonstrate that artificially re-regulating replication factor expression from the genome can produce stable plasmid copy numbers, that phenotype varies with copy number, and that DIAL strains can accelerate development of genetic devices.

\section{Results and Discussion}

\section{ColE2 as a Trans-Activated Origin}

A previous report [18] identified a minimal 32 bp region of ColE2 sufficient to support replication of a plasmid when repA is provided in trans. We first recapitulated this behaviour by transforming a plasmid bearing the ColE2 minimal origin (pBjk2164-jtk2619) into cells constitutively expressing the trans-acting repA gene from a plasmid (MC1061 + pBca9145-jtk2627). Although we observed transformants, they exhibited small colony morphologies. Presuming this observation to reflect plasmid instability (as suggested by data in [19]), we repeated the experiment using a larger 470 bp fragment of the ColE2 plasmid as the origin (pBjk2164-jtk2642) in the hope that any context dependent influence on the minimal origin would be eliminated, or that non-essential factors contributing to robustness would be captured. This yielded colonies with morphologies indistinguishable from untransformed cells (data not shown).

\section{Orthogonality of R6K and ColE2}

We next examined if ColE2 and R6K were orthogonal origins of replication. Plasmids bearing either an R6K or ColE2 origin of replication were transformed or cotransformed into cells expressing pir, $\operatorname{rep} A$, or both. We observed colonies only when a plasmid was transformed into cells possessing the cognate replication factor, as expected.

\section{Construction of DIAL Strains}

To generate cells expressing diverse levels of the transacting factors Pir and RepA, we integrated expression cassettes with randomized ribosome binding sites (RBSs) into the genome, thereby creating strain sets JTK160 and JTK164 for pir and $\operatorname{repA}$, respectively. We subsequently visualized copy number variation by transforming the libraries with reporter plasmids constitutively expressing sfGFP [20] (pBjk2741-jtk2828 or pBjk2807jtk2828). After a preliminary fluorescence analysis of 380 clones of each type (data not shown), 24 were selected for further investigation. After a second round of fluorescence measurement, 10 variants of each type that spanned the range of observed fluorescence levels were chosen for full characterization. The sequences of the selected RBSs are reported in table 1.

\section{Characterization of DIAL Strains: Copy Number, Cell-to- Cell Variability, and Stability}

We characterized three important properties of plasmids in the DIAL strains: copy number, cell-to-cell variation, and stability. To estimate the copy number supported by each strain, we employed qPCR to examine plasmid content both at mid log and at stationary phase (Figure 1). Based on this analysis, a ColE2 plasmid in the DIAL strains spans the range of $\sim 1-60$ copies per genome equivalent, while an R6K plasmid in the DIAL strains spans the range of $\sim 5-250$ copies per genome equivalent. This covers nearly the entire range of

Table 1 RBSs of repA and pir expression variants

\begin{tabular}{|c|c|c|}
\hline Strain & Variant & Sequence \\
\hline \multirow[t]{10}{*}{ JTK160 (repA) } & A & TCTAGAATACCTGATG \\
\hline & B & GTGAGAAGTGAACGTG \\
\hline & C & CCGAGAACGTAGGATG \\
\hline & $D$ & GGCAGAAAGTTGAATG \\
\hline & $E$ & GTGAGAAAGCTCTGTG \\
\hline & $\mathrm{F}$ & CTCAGAACCGAATATG \\
\hline & G & GTGAGAATGCTITATG \\
\hline & $\mathrm{H}$ & TTGAGAAAAACAGATG \\
\hline & । & GGGAGAAAGATGGGTG \\
\hline & J & GGGAGAAAACAAAATG \\
\hline \multirow[t]{10}{*}{ JTK164 (pir) } & $A$ & GCTGGAACAGGTGGTG \\
\hline & B & TAAGGAATTAGGTGTG \\
\hline & C & GGGGGAAGGGCATGTG \\
\hline & $\mathrm{D}$ & TTGGGAACAATTCATG \\
\hline & $E$ & TCCGGAAGACTAGGTG \\
\hline & $\mathrm{F}$ & GTCGGAAAGGGCT $\overline{\underline{G T G}}$ \\
\hline & G & GTGGGAATAGAATATG \\
\hline & $\mathrm{H}$ & ACGGGAATGTAACGTG \\
\hline & । & ACTGGAACTGCATGTG \\
\hline & J & ATCGGAAACATAGGTG \\
\hline
\end{tabular}

Start codons are underlined. 


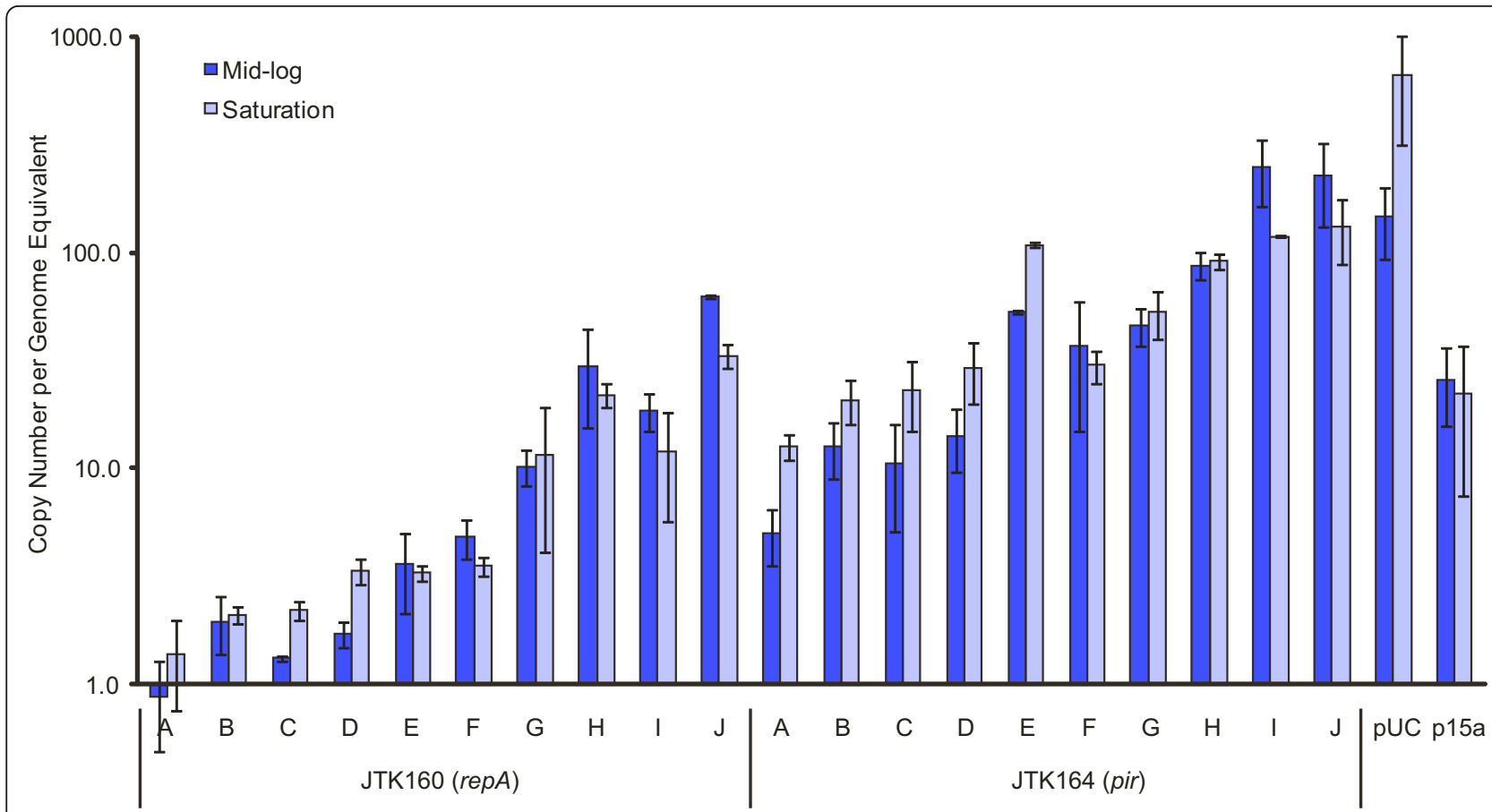

Figure 1 qPCR estimation of plasmid copy number. JTK160 (repA) and JTK164 (pir) variants were transformed with pBjk2992-jtk2541 and pBjk2993-jtk2541, respectively. JTK160J and JTK164J were also transformed with pBjo1601A-jtk2541 (p15a origin) and pBjk3057-jtk2541 (pUC origin). Two biological replicates of each sample were prepared and analyzed using qPCR. Error bars represent standard deviation.

reported plasmid copy numbers, from single copy to nearly pUC levels. We observed that the pUC plasmid exhibited 4-5 fold increased copy numbers at stationary phase, while the p15a, R6K, and ColE2 plasmids showed $\sim 2$-fold or lower changes.

We next examined sfGFP expression in samples of each of the cells by flow cytometry (Figure 2) to determine cell-to-cell variability. The ColE2 and R6K plasmids generally exhibit similar distributions to $\mathrm{p} 15 \mathrm{a}$ and pUC origins. Strains JTK160I and JTK164E, which have mean expression levels within $25 \%$ of p15a levels, have coefficients of variance that fall within $25 \%$ of p 15 a levels. Similarly, JTK160J and JTK164I, which have mean expression levels within $25 \%$ of pUC levels, have coefficients of variance that fall within $25 \%$ of pUC levels. It is unclear if the relatively high coefficients of variance for JTK160A and JTK164A are a result of noise at low fluorescence (as evidenced by the very high MC1061 coefficients of variance) or due to true variance in plasmid copy number or GFP expression.

Finally, we monitored the stability of plasmids in midlevel expression variants of both pir and repA after 100 generations without selection (Figure 3). Both pir and repA variants exhibited high stability, losing the plasmid in only $5.2 \%$ or $0.5 \%$ of cells, respectively. These numbers fall between the stability of the control p15a and pUC plasmids, which lost the plasmid in $23.5 \%$ or $.25 \%$ of cells, respectively.

\section{Optimization of Violacein Expression}

As a simple demonstration of the utility of the DIAL strains, we optimized the expression level of the violacein biosynthesis operon, VioABCDE. While moderate production levels of the deeply purple metabolite are tolerated in E. coli, high levels can be toxic or cause instability [17]. We cloned the operon behind both a weak and a strong constitutive promoter to illustrate the flexibility afforded by controlling copy number. Figure 4 shows the colonies that result from transforming both weakly and strongly expressed operons (jtk3070 and jtk3080, respectively) into all of the DIAL strains. Production of violacein, as indicated by purple coloration, clearly increases as copy number increases, up to some threshold level. Beyond that threshold, colonies begin to grow at reduced rates or not at all. The large colonies in the high copy strains (JTK160G, JTK160I, and JTK164H) with a strong promoter were sequenced and confirmed to be escape mutants in which a fragment of the strong constitutive promoter has recombined out, demonstrating the risk of overstressing the cells.

\section{Conclusions}

We have developed and characterized two sets of strains that support the R6K and ColE2 origin of replication at a wide range of different copy numbers enabling rapid exploration of gene and circuit dosage. To accomplish this, we placed the trans-acting replication factors from 


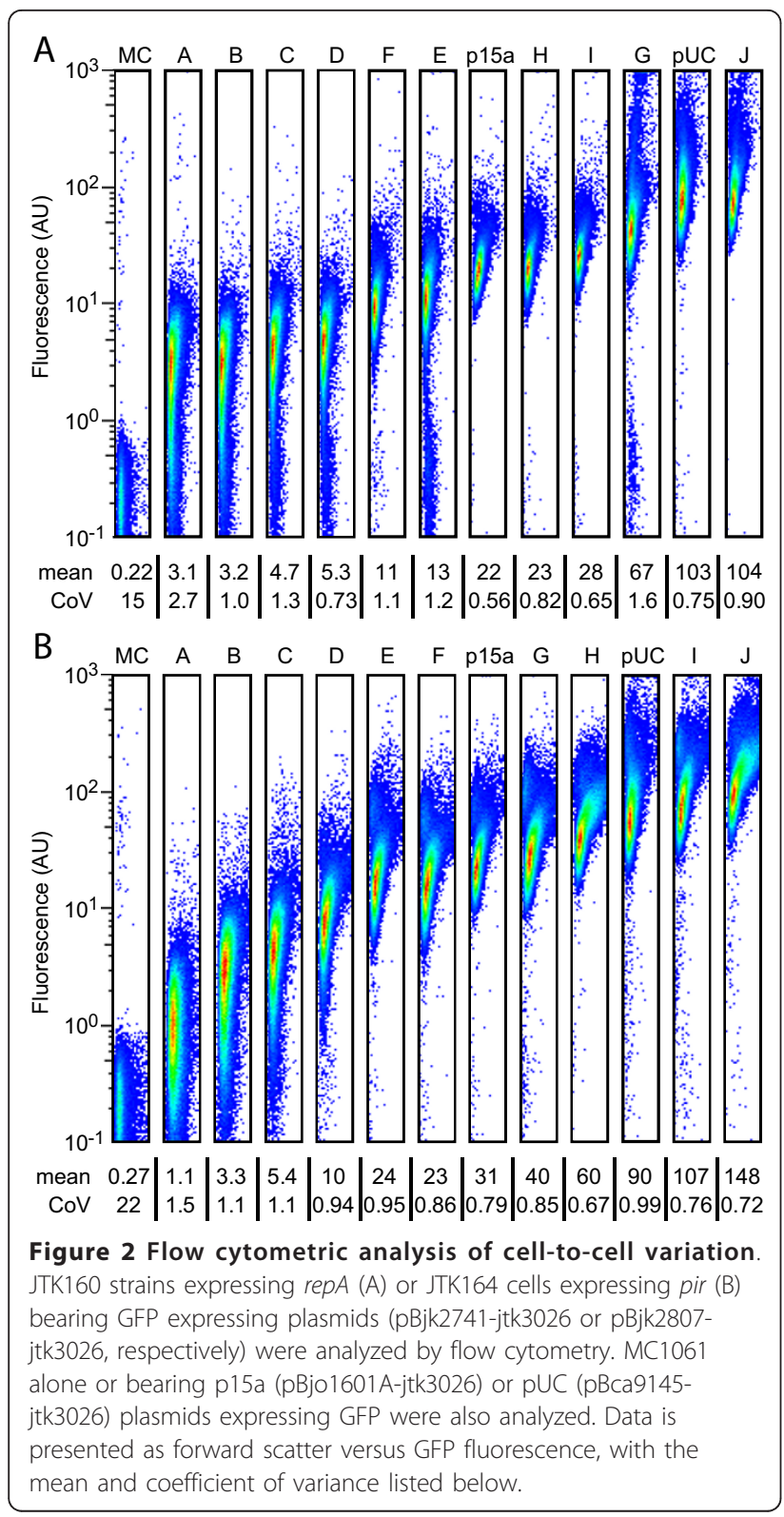

each replicon under artificial transcriptional regulation in the genome, leaving only the origins of replication on the plasmids themselves. Although negative feedback relying on elements $5^{\prime}$ of the trans-acting factor open reading frame has been implicated as one factor helping to maintain stable copy numbers in both ColE2 [21] and R6K [14], we found that engineered cells stably maintained plasmid copy numbers despite removal of all 5' regulatory elements. This is consistent with the existence of additional feedback mechanisms, as has been suggested for both R6K [22] and ColE2 [23].

To generate copy number diversity in the DIAL strains, we created a library of RBS variants of the trans-acting replication factor in the genome. Although

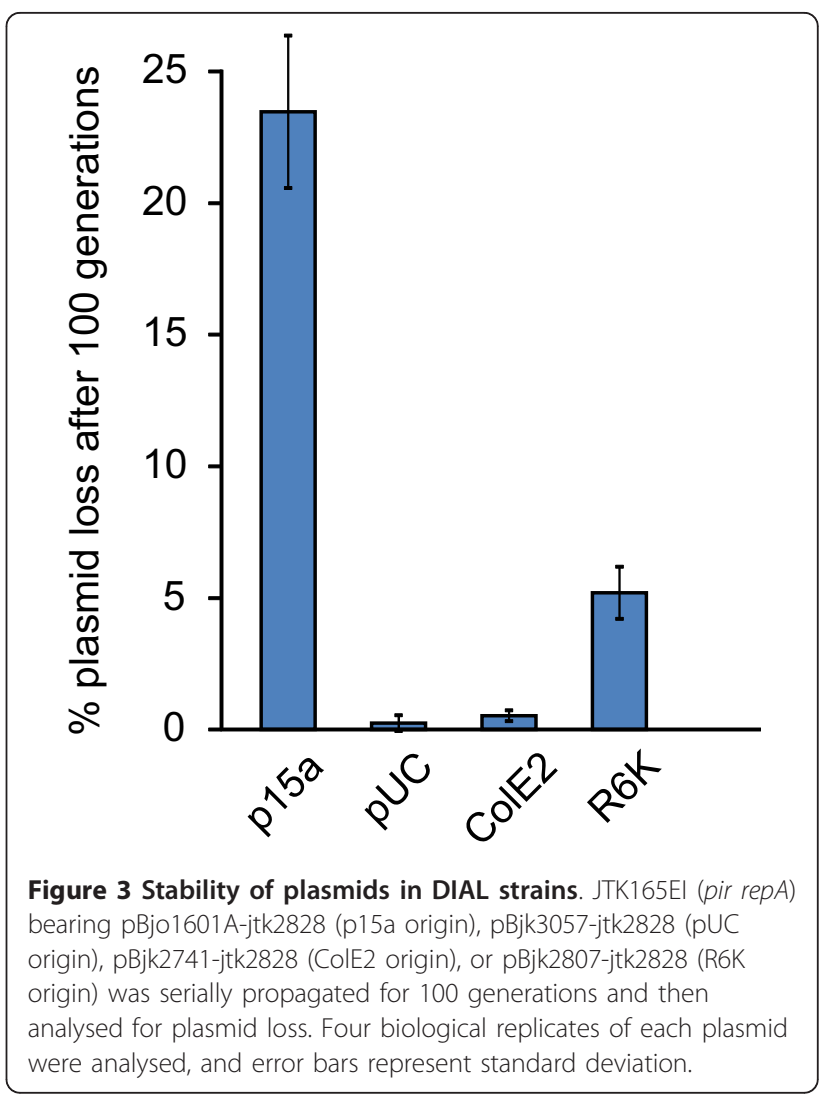

other strategies, such as the use of an inducible promoter or a library of promoters, could also have achieved diverse levels of trans-acting factor expression, varying the RBS enables compatibility with genetic circuits employing any promoter and maintains a consistent noise profile across strains due to stochastic transcription effects [24]. We employed a novel mechanism of generating RBS libraries in the genome: lambda red based integration of Splicing by Overlap Extension (SOEing) [25] PCR products. Multiplex automated genome engineering [26] has also been employed for creating libraries of modified genomic RBSs. While that process is a powerful method for modifying genes already in the genome, this work required simultaneous modification and introduction into the genome of an exogenous gene. In such cases, PCR based integration is an excellent option for library construction, particularly where a relatively small number of variants $(<10,000)$ is sufficient to isolate the desired functionality.

The DIAL strains are the first tool capable of systematically varying genetic circuit dosage without altering the local genetic context. Previous studies examining the impact of circuit dosage in prokaryotes have been largely theoretical (e.g. [27,28]), and in eukaryotes focus only on low ( 1-2) copy numbers (e.g. [29]). Because the theoretical predictions suggest that circuit dosage 


\section{JTK160/JTK164}

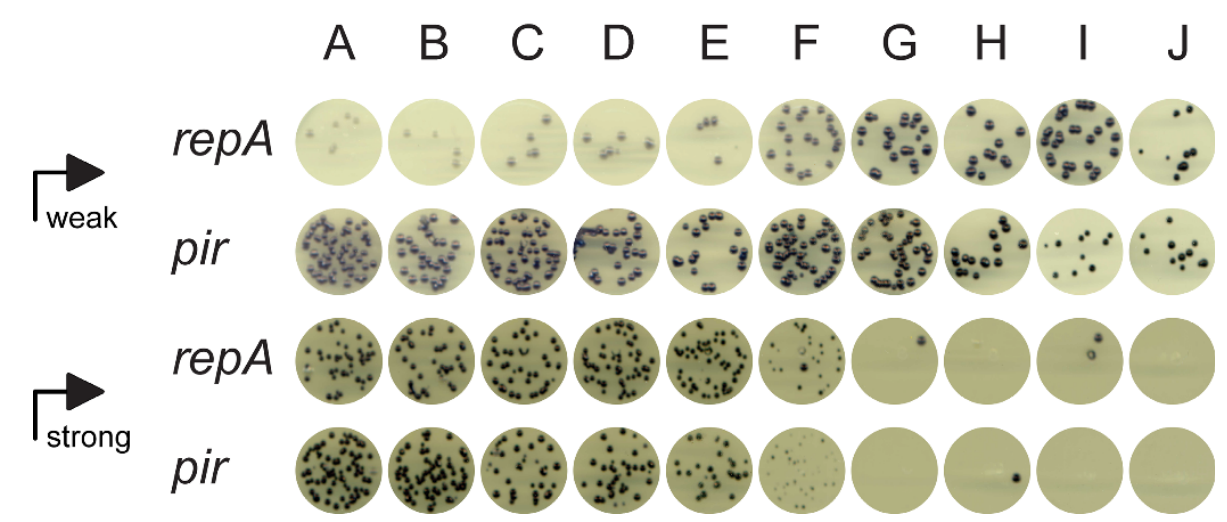

Figure 4 Optimization of violacein production. Variants of JTK160 (repA) or JTK164 (pir) were transformed with plasmids containing either a weak promoter (pBjk2741-jtk3070 or pBjk2807-jtk3070) or strong promoter (pBjk2741-jtk3080 or pBjk2807-jtk3080) driving expression of the violacein synthesis pathway. Transformants were plated as $5 \mu \mathrm{l}$ spots. Small circles for a given strain set/plasmid combination are cropped from the same original plate image taken on a flatbed scanner. Gradient indicates the trend of copy number in strain variants.

has a significant impact on the function of some genetic circuits, it is important to empirically verify the robustness or fragility of different circuit architectures. Using the DIAL strains, network behaviour and expression noise can be rapidly assessed at a wide variety of different circuit dosages.

Of great practical use, the DIAL strains offer a rapid, facile mechanism for determining desired expression levels, making it a tool with broad applicability in genetic engineering. The trivial operation of transforming the same plasmid into different strains is sufficient to provide information on the maximum tolerated expression level for a given protein, pathway, or circuit, and screening of viable colonies reveals the optimal expression level for a desired phenotype. We demonstrated this simple capability by optimizing expression of the violacein biosynthesis pathway, which in excess produces moderate toxicity in E. coli. Regardless of whether that starting point was a weakly or a strongly expressed operon, deeply purple yet healthy cells were isolated when matched with the appropriate strain. Knowing the optimal gene dosage can be leveraged to change the context of a gene or operon without altering the phenotype. Since the copy number is known, any change in protein dosage resulting from changing the context of the system (such as by integration into the genome) can be compensated for by using existing tools such as the RBS calculator [7] or a set of standard promoters [5].

Importantly, the ColE2 and R6K origins are orthogonal and can co-exist in the same cell, and the two sets of DIAL strains were designed to enable ready combination of both trans factors into a single strain by P1 transduction. Having a single set of cells with both orthogonal origins allows both the relative and absolute levels of two genes or sets of genes to be optimized by, for example, co-transformation into a pool of competent cells. Although R6K has already seen widespread use because of its ability to split into trans and cis elements, having a variety of copy number variants available for both R6K and ColE2 provides an even more powerful toolset for expression level optimization and balancing, circuit dosage investigation, and novel selection schemes.

\section{Methods \\ Media}

Strains were propagated in LB broth and LB agar plates, with addition of $100 \mu \mathrm{g} / \mathrm{ml}$ ampicillin sodium salt, 50 $\mu \mathrm{g} / \mathrm{ml}$ spectinomycin dihydrochloride pentahydrate, 25 $\mu \mathrm{g} / \mathrm{ml}$ kanamycin sulphate, and/or $10 \mu \mathrm{g} / \mathrm{ml}$ trimethoprim if appropriate.

\section{Plasmids}

Plasmids were constructed using BglBrick standard assembly [30]. Full sequences of plasmids are available in Additional file 1, Table S1. The replicon of ColE2-P9 [16] is referred to as ColE2 for convenience. The gamma origin of $\mathrm{R} 6 \mathrm{~K}$ is similarly referred to simply as the R6K origin.

\section{Genomic RBS Library Construction}

Template plasmids pBjk2648r-jtk2951 and pBjk2741jtk3041, illustrated schematically in Figure 5, were first constructed. Splicing by overlap extension SOEing PCR [25] with degenerate oligos (table 2) was used to 


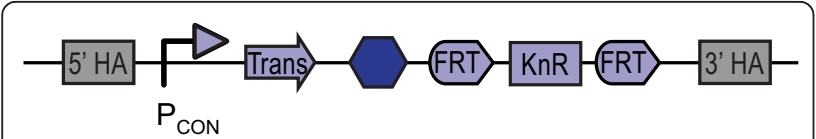

Figure 5 Schematic of templates constructed for genomic integration. Plasmids pBjk2648r-jtk2951 and pBjk2741-jtk3041 both contain: 1) a $\sim 500$ bp homology arm (HA) matching the $5^{\prime}$ end of the genomic insertion site 2) a constitutive promoter $\left(\mathrm{P}_{\mathrm{CON}}\right)$ 3) the ORF of the trans-acting replication factor (Trans) 4) a terminator 5) a kanamycin resistance cassette (KnR) flanked by FRT sites and 6) a $\sim 500$ bp HA matching the $3^{\prime}$ end of the genomic insertion site. Full sequences can be found in Additional file 1, Table S1.

generate RBS variants (NNNGGAANNNNNNRTG for pir and NNNAGAANNNNNNRTG for repA) of the cassettes on the template plasmids. The final PCR products were gel purified using Zymo columns according to the manufacturer's instructions, and then used to modify the genome of strain MC1061 [31] by the procedure of Datsenko and Wanner [32]. The resulting libraries consisted of 10,000 members each, and were pooled before preliminary transformation with fluorescent protein expressing plasmids and analysis of fluorescence levels. Variants ultimately selected for full characterization were P1 transduced into MC1061 cells before further analysis to eliminate the initial fluorescent plasmid.

\section{Plasmid Copy Number Estimation by qPCR}

Plasmid copy number per genome equivalent was estimated using the relative quantitation method described previously [33]. Briefly, cells were subcultured 1:100 into fresh media and grown until mid-log or stationary phase before total DNA isolation using QIAamp DNA Mini kits (Qiagen) according to the manufacturer's instructions. DNA samples and 10-fold serial dilutions of a purified calibrator plasmid bearing a single copy of both bla and $d x s$ (pBca9145-jtk3068) were then amplified on an iCycler with iQ5 real-time PCR detection system (Biorad)

Table 2 Oligos used for library construction

\begin{tabular}{|c|c|c|}
\hline \multirow[t]{5}{*}{ pir } & pBjk2741-jtk3041 & $5^{\prime}-3^{\prime}$ oligos \\
\hline & Outer oligo F & CACATGGCGACCAGATCAATAC \\
\hline & Outer oligo R & ATTGCCGCAGGTGGAAAC \\
\hline & Inner oligo F & $\begin{array}{l}\text { GGTACAGTGCTAGCGGATCTNNNGGAA } \\
\text { NNNNNNRTGAGACTCAAGGTCATGATGG }\end{array}$ \\
\hline & Inner oligo R & GATCCGCTAGCACTGTACC \\
\hline \multirow[t]{5}{*}{ repA } & pBjk2648r-jtk2951 & $5^{\prime}-3^{\prime}$ oligos \\
\hline & Outer oligo F & GGATTTTCCTTGTTTCCAGAG \\
\hline & Outer oligo $\mathrm{R}$ & GCTTACGGCTTTATATTACGGG \\
\hline & Inner oligo F & $\begin{array}{l}\text { CTCGTCAGTAACGAGCCCTNNNAGAAN } \\
\text { NNNNNRTGAGCGCCGTACTTC }\end{array}$ \\
\hline & Inner oligo R & AGGGCTCGTTACTGACGAG \\
\hline
\end{tabular}

using previously validated primer pairs [33] for both bla and $d x s$ (bla F: 5'-CTACGATACGGGAGGGCTTA-3' blaR: 5'-ATAAATCTGGAGCCGGTGAG-3' dxsF: 5'CGAGAAACTGGCGATCCTTA-3' dxsR: 5'-CTTCATCAAGCGGTTTCACA-3'). Each reaction contained 25 $\mu \mathrm{l}: 12.5 \mu \mathrm{l}$ Absolute QPCR SYBR Green Fluorescein Mix (Thermo Scientific), $1.25 \mu \mathrm{l}$ each primer $(10 \mu \mathrm{M}), 3.75 \mu \mathrm{l}$ $\mathrm{H}_{2} \mathrm{O}$, and $5 \mu \mathrm{l}$ sample DNA. Reaction conditions were as follows: an initial denaturation at $95^{\circ} \mathrm{C}$ for 10 minutes, followed by 40 cycles of $95^{\circ} \mathrm{C}$ for 10 seconds, $63^{\circ} \mathrm{C}$ for 15 seconds, and $72^{\circ} \mathrm{C}$ for 15 seconds. Measurements were taken at the end of each extension step. Copy numbers were calculated by using the calibrator standard curves to determine the quantity of plasmid (bla) and genome $(d x s)$ dna for a given sample in arbitrary units, and then calculating their ratio.

\section{Flow Cytometry}

Cells grown to stationary phage were subcultured 1:100 in fresh media, grown until mid-log, resuspended in PBS, and then examined on a Coulter Epics Xl-MCl instrument with a $488 \mathrm{~nm}$ excitation wavelength and $525 \mathrm{~nm}$ emission bandpass filter.

\section{Stability Analysis}

Single colonies were picked and grown to stationary phase under selection. Cells were then subcultured 1:10 ${ }^{6}$ and grown back to stationary phase without selection, which corresponds to 20 generations of growth. The dilution and regrowth was repeated serially for 100 generations, at which point dilutions of cells were plated on non-selective media, and colonies were examined for sfGFP fluorescence as an indicator of plasmid presence.

\section{Additional material}

Additional file 1: Lists the full sequences of plasmids and strain modifications used in this study.

\section{Acknowledgements}

The authors would like to thank Dr. Tateo Itoh for providing the ColE2-P9 replicon. This work was supported by the National Science Foundation Synthetic Biology Engineering Research Center (SynBERC). JTK was supported by a National Science Foundation Graduate Research Fellowship.

\section{Author details}

'Department of Bioengineering, University of California, Berkeley, USA.

${ }^{2}$ Department of Bioengineering, University of California, San Diego, USA. ${ }^{3}$ Berkeley National Laboratory, Physical Biosciences Division; QB3: California Institute for Quantitative Biological Research; 327 Stanley Hall, Berkeley, CA 94720, USA.

\section{Authors' contributions}

JTK and JCA designed experiments. JTK and SC carried out experiments. JTK and JCA drafted the manuscript. All authors read and approved the final manuscript. 


\section{Competing interests}

The authors declare that they have no competing interests.

Received: 30 April 2011 Accepted: 25 July 2011 Published: 25 July 2011

\section{References}

1. Jones Prather KL: Low-Copy Number Plasmids as Artificial Chromosomes. In The metabolic pathway engineering handbook: tools and applications. Edited by: Smolke CD. Boca Raton: CRC Press; 2010:

2. Humphreys DP, Carrington B, Bowering LC, Ganesh R, Sehdev M, Smith BJ, King LM, Reeks DG, Lawson A, Popplewell AG: A plasmid system for optimization of Fab' production in Escherichia coli: importance of balance of heavy chain and light chain synthesis. Protein Expr Purif 2002, 26:309-320.

3. Kim Y, Wang X, Zhang XS, Grigoriu S, Page R, Peti W, Wood TK: Escherichia coli toxin/antitoxin pair MqsR/MqsA regulate toxin CspD. Environ Microbiol 2010, 12:1105-1121.

4. Arkin A, Ross J, McAdams HH: Stochastic kinetic analysis of developmental pathway bifurcation in phage lambda-infected Escherichia coli cells. Genetics 1998, 149:1633-1648.

5. Davis JH, Rubin AJ, Sauer RT: Design, construction and characterization of a set of insulated bacterial promoters. Nucleic Acids Res 2011, 39:1131-1141.

6. Smolke CD, Carrier TA, Keasling JD: Coordinated, differential expression of two genes through directed mRNA cleavage and stabilization by secondary structures. Appl Environ Microbiol 2000, 66:5399-5405.

7. Salis HM, Mirsky EA, Voigt CA: Automated design of synthetic ribosome binding sites to control protein expression. Nat Biotechnol 2009, 27:946-950.

8. Tyo KE, Ajikumar PK, Stephanopoulos G: Stabilized gene duplication enables long-term selection-free heterologous pathway expression. Nat Biotechnol 2009, 27:760-765.

9. Chew LC, Tacon WC: Simultaneous regulation of plasmid replication and heterologous gene expression in Escherichia coli. J Biotechnol 1990, 13:47-60.

10. Togna AP, Shuler ML, Wilson DB: Effects of plasmid copy number and runaway plasmid replication on overproduction and excretion of betalactamase from Escherichia coli. Biotechnol Prog 1993, 9:31-39.

11. Larsen JE, Gerdes K, Light J, Molin S: Low-copy-number plasmid-cloning vectors amplifiable by derepression of an inserted foreign promoter. Gene 1984, 28:45-54.

12. Wild J, Hradecna Z, Szybalski W: Conditionally amplifiable BACs: switching from single-copy to high-copy vectors and genomic clones. Genome Res 2002, 12:1434-1444

13. Preston A: Choosing a cloning vector. Methods Mol Biol 2003, 235:19-26.

14. Shafferman A, Kolter R, Stalker D, Helinski DR: Plasmid R6K DNA replication. III. Regulatory properties of the pi initiation protein. J Mol Biol 1982, 161:57-76.

15. Metcalf $W W$, Jiang $W$, Wanner BL: Use of the rep technique for allele replacement to construct new Escherichia coli hosts for maintenance of R6K gamma origin plasmids at different copy numbers. Gene 1994, 138:1-7.

16. Horii T, Itoh T: Replication of ColE2 and ColE3 plasmids: the regions sufficient for autonomous replication. Mol Gen Genet 1988, 212:225-231.

17. Ahmetagic A, Pemberton JM: Stable high level expression of the violacein indolocarbazole anti-tumour gene cluster and the Streptomyces lividans amyA gene in E. coli K12. Plasmid 2010, 63:79-85.

18. Yasueda H, Horii T, Itoh T: Structural and functional organization of ColE2 and ColE3 replicons. Mol Gen Genet 1989, 215:209-216.

19. Hunger M, Schmucker R, Kishan V, Hillen W: Analysis and nucleotide sequence of an origin of DNA replication in Acinetobacter calcoaceticus and its use for Escherichia coli shuttle plasmids. Gene 1990, 87:45-51.

20. Pedelacq JD, Cabantous S, Tran T, Terwilliger TC, Waldo GS: Engineering and characterization of a superfolder green fluorescent protein. Nat Biotechnol 2006, 24:79-88.

21. Sugiyama T, Itoh T: Control of ColE2 DNA replication: in vitro binding of the antisense RNA to the Rep mRNA. Nucleic Acids Res 1993, 21:5972-5977.

22. Kunnimalaiyaan S, Inman RB, Rakowski SA, Filutowicz M: Role of pi dimers in coupling ("handcuffing") of plasmid R6K's gamma ori iterons. J Bacteriol 2005, 187:3779-3785.
23. Shinohara $M$, Itoh $T$ : Specificity determinants in interaction of the initiator (Rep) proteins with the origins in the plasmids ColE2-P9 and ColE3-CA38 identified by chimera analysis. J Mol Biol 1996, 257:290-300.

24. Tyagi S, Genomics: E. coli, what a noisy bug. Science 329:518-519.

25. Horton RM, Cai ZL, Ho SN, Pease LR: Gene splicing by overlap extension: tailor-made genes using the polymerase chain reaction. Biotechniques 1990, 8:528-535.

26. Wang HH, Isaacs FJ, Carr PA, Sun ZZ, Xu G, Forest CR, Church GM: Programming cells by multiplex genome engineering and accelerated evolution. Nature 2009, 460:894-898.

27. Mileyko Y, Joh RI, Weitz JS: Small-scale copy number variation and largescale changes in gene expression. Proc Natl Acad Sci USA 2008, 105:16659-16664.

28. Loinger A, Biham O: Analysis of genetic toggle switch systems encoded on plasmids. Phys Rev Lett 2009, 103:068104

29. Acar M, Pando BF, Arnold FH, Elowitz MB, van Oudenaarden A: A general mechanism for network-dosage compensation in gene circuits. Science 2010, 329:1656-1660

30. Anderson JC, Dueber JE, Leguia M, Wu GC, Goler JA, Arkin AP, Keasling JD, BglBricks: A flexible standard for biological part assembly. Journal of biological engineering 2010, 4:1.

31. Casadaban MJ, Cohen SN: Analysis of gene control signals by DNA fusion and cloning in Escherichia coli. J Mol Biol 1980, 138:179-207.

32. Datsenko KA, Wanner BL: One-step inactivation of chromosomal genes in Escherichia coli K-12 using PCR products. Proc Natl Acad Sci USA 2000, 97:6640-6645.

33. Lee C, Kim J, Shin SG, Hwang S: Absolute and relative QPCR quantification of plasmid copy number in Escherichia coli. J Biotechnol 2006, 123:273-280.

doi:10.1186/1754-1611-5-10

Cite this article as: Kittleson et al:: Rapid optimization of gene dosage in E. coli using DIAL strains. Journal of Biological Engineering 2011 5:10.

\section{Submit your next manuscript to BioMed Central and take full advantage of:}

- Convenient online submission

- Thorough peer review

- No space constraints or color figure charges

- Immediate publication on acceptance

- Inclusion in PubMed, CAS, Scopus and Google Scholar

- Research which is freely available for redistribution
C Biomed Central 